\title{
Pedagogías para la paz, la relevancia de la perspectiva de géneros y la interseccionalidad
}

\author{
Pedagogies for peace, the relevance of the gender \\ approach and the intersectionality \\ Pedagogias para a paz, a relevância da perspectiva \\ de gêneros e a interseccionalidade
}

Luisa Amézquita Aguirre ${ }^{1}$
Universidad Pedagógica y Tecnológica de Colombia
https://orcid.org/0000-0001-7480-7614
Celina Trimiño Velásquez ${ }^{2}$
Universidad Pedagógica y Tecnológica de Colombia, Colombia $_{\text {https://orcid.org/0000-0002-6786-4378 }}$

Recepción: 30/01/2020

Evaluación: 13/05/2020

Aceptación: 28/05/2020

Artículo de Investigación - Reflexión

https://doi.org/10.19053/01227238.11918

\section{RESUMEN}

El objetivo de este artículo es indagar algunos desarrollos de las pedagogías para la paz y su articulación con teorías que impugnan al patriarcado. Su originalidad radica en conectar la disertación sobre las pedagogías para la paz, la relevancia de la perspectiva de géneros y la interseccionalidad, e impele a que se desborden los límites de epistemologías excluyentes, para avanzar hacia propuestas alternativas. El método cualitativo-interpretativo facilita emplear estrategias de análisis documental para examinar teorías que contribuyen, con otros presupuestos, a evidenciar la diversidad de los seres humanos y a denunciar las múltiples opresiones de las que son objeto sectores de la población por distintas causas que pretenden justificar su exclusión de los espacios públicos y decisorios, lo que viola sus derechos humanos, y en varios países, lleva incluso, a su eliminación física. Esta dinámica de exploración teórica permite concluir que, aproximarse a las pedagogías para la paz, es un ejercicio permanente que involucra tanto a los currículos educativos como a las acciones de la vida cotidiana, quehacer que exige un esfuerzo mancomunado de la sociedad colombiana para la consolidación de una paz estable y duradera.

Palabras clave: Pedagogías para la paz; género; interseccionalidad; Colombia.

1 Doctora en Ciencias de la Educación - Especialidad en Pedagogía Escolar de la Università Pontificia Salesiana di Roma (Italia). Docente de la Escuela de Educación Infantil, Facultad de Ciencias de la Educación, Universidad Pedagógica y Tecnológica de Colombia. Grupo de Investigación Feminismos, Géneros y Derechos Humanos (GIEPEG), correo electronico: luisa.amezquita@ uptc.edu.co; infanciasyexpresiones@uptc.edu.co

2 Doctora en Derechos Fundamentales de la Universidad Carlos III de Madrid (España). Docente de la Escuela de Ciencias Sociales, Facultad de Ciencias de la Educación, Universidad Pedagógica y Tecnológica de Colombia. Investigadora Grupo de Investigación Feminismos, Géneros y Derechos Humanos (GIEPEG), correo electronico: celina.trimino@uptc.edu.co; grupo.giepeg@uptc.edu.co 


\section{ABSTRACT}

The objective of this article is to research some developments in pedagogies for peace and their joint with theories that challenge patriarchy. Its originality lies on connecting the dissertation of pedagogies for peace, the relevance of the gender approach and intersectionality that impels the limits of exclusive epistemologies to be overflowed, to move towards alternative proposals. The qualitative-interpretative method helps the use of documentary analysis strategies to analyze the theories that contribute with other assumptions to highlight the diversity of human beings and to denounce the multiple oppressions that different community spaces are subjected for different reasons, which lead to trying to justify their exclusion from public and decision-making spaces, and in several countries even to their physical removal, which violates their human rights. This dynamic of theoretical exploration allows us to conclude that approaching pedagogies for peace is a permanent exercise that involves both educational curriculum and actions of our daily life, a task that requires a joint effort from the whole of Colombian society for the consolidation of a stable and lasting peace.

Key words: Pedagogies for peace; gender; intersectionality; Colombia.

\section{RESUMO}

O objetivo deste artigo é indagar alguns desenvolvimentos das pedagogias para a paz e sua articulação com teorias que impugnam o patriarcado. Sua originalidade radica em conectar a dissertação sobre as pedagogias para a paz, a relevância da perspectiva de gêneros e a interseccionalidade e impede a que ultrapassem os limites de epistemologias excludentes, para avançar para propostas alternativas. O método qualitativo-interpretativo facilita empregar estratégias de análise documental para examinar teorias que contribuem com outros pressupostos para evidenciar a diversidade dos seres humanos e a denunciar as múltiplas opressões de que são objeto setores da população por diferentes causas, que levam a propor a justificativa de sua exclusão dos espaços públicos e decisórios, e em vários países, incluída a sua eliminação física, o que viola seus direitos humanos. Esta dinâmica de exploração teórica permite concluir que aproximar-se das pedagogias para a paz é um exercício permanente que envolve tanto os currículos educativos como as ações da vida cotidiana, uma ação que exige um esforço mancomunado do conjunto da sociedade colombiana para a consolidação de uma paz estável e duradoura.

Palavras-chave: Pedagogias para a paz; gênero; interseccionalidade; Colômbia.

\section{INTRODUCCIÓN}

Este artículo busca indagar el desarrollo de las pedagogías para la paz y la cada vez mayor trascendencia de la perspectiva de género(s) y su entronque con la interseccionalidad. Este ejercicio investigativo, realizado desde un enfoque cualitativo-interpretativo, muestra que, aunque se han incrementado las investigaciones y los escritos sobre la paz, todavía son limitados aquellos que se soportan en teorías que involucren otros puntos de vista, provenientes de distintos sectores de la sociedad que resalten la relevancia de incorporar conocimientos que no solo cuestionen el androcentrismo, sino que también van más 
allá, teniendo en cuenta diferentes historias, contextos y diversidad. Es así como se torna necesario ampliar la mirada conceptual, escudriñar las experiencias desde abajo en un "diálogo de saberes" y, en consecuencia, explorar propuestas alternativas. Estos elementos pueden coadyuvar a proseguir el debate sobre estas problemáticas en el interior de los currículos y fuera de estos; de igual forma, es ineludible preguntarse por la situación de amplios grupos sociales que sistemáticamente ven vulnerados sus derechos humanos por las profundas desigualdades subyacentes en las estructuras socioeconómicas y políticas. No cabe duda de que Colombia está en mora de asumir acciones educativas concretas en todos los niveles, para afrontar las condiciones de la población que por décadas ha vivido inmersa en entornos de conflicto armado - en medio, de un lado o del otro- que impregnan la vida cotidiana tanto en los ámbitos públicos como privados y familiares.

La originalidad se traduce en el análisis entrelazado sobre las pedagogías para la paz, la relevancia de la perspectiva de géneros y la interseccionalidad, contribuyendo a la búsqueda de caminos teóricamente fundamentados y en prácticas concretas. En este marco, se realiza una aproximación a otras posibilidades pedagógicas que propendan por acercar al estudiantado de los diferentes niveles educativos, y a la sociedad en general, a pensar con seriedad en el respeto a todos los seres humanos y en la responsabilidad que entraña la construcción de la paz. Asimismo, se reconocen las experiencias y valiosos aportes de las mujeres en estos procesos de paz.

\section{Pedagogías para la paz}

Las experiencias en varios países muestran que, en el marco de los derechos humanos y la paz, adquieren trascendental importancia la educación y la pedagogía para la paz ${ }^{3}$. Desde esta óptica, se toma como ejemplo cercano a Colombia, con una larga historia de conflictos violentos, entre ellos el armado, además por ser uno de los países más desiguales de América Latina y el Caribe, razón por la cual también se debe trabajar conjuntamente para transformar las realidades de muchas personas excluidas por diversas razones. En este caso, hay que hacer hincapié en que "la etapa del posacuerdo implica un gran esfuerzo y el compromiso de toda la sociedad. Se debe consolidar la reconstrucción del tejido social, lo que seguramente abarcará a más de una generación" ${ }^{\prime 4}$.

Aquí, toman valor las cavilaciones que giran alrededor de ideas que permiten examinar los conflictos, la posibilidad del diálogo y las vías no violentas, el conocimiento de los derechos humanos, las concepciones sobre la paz: su caracterización y tendencias. Justamente, el derecho a la paz inspira el sentido de una

3 "Los emergentes modelos pedagógicos de la UPTC deben incorporar la cultura de la paz como su acicate central". Diana Elvira Soto Arango, José Pascual Mora García y José Rubens Lima Jardilino, "Formación de docentes y modelo pedagógico en la Universidad Pedagógica y Tecnológica de Colombia”, Revista Historia de la Educación Latinoamericana vol. 19, n. ${ }^{\circ} 29$ (2017): 38.

4 Celina Trimiño Velásquez y Luisa Amézquita Aguirre, "Reflexiones desde la universidad sobre educación en derechos humanos y para la paz", Revista Historia de la Educación Latinoamericana vol. 20, n. ${ }^{\circ} 31$ (2018):111. 
cultura de paz que, desde una pedagogía dialogante, contribuya a la generación y desarrollo de propuestas mancomunadas; puesto que "la acción política junto a los oprimidos, en el fondo, debe ser una acción cultural para la libertad, y por ello mismo, una acción con ellos ${ }^{\prime \prime}$, lo que vislumbra caminos que pueden recorrer todas las personas. Esta opinión se construye y fortalece en la educación que prioriza el pensamiento crítico emancipatorio, dado que, cuando una persona está empoderada, es capaz de manifestar su inconformidad ante actitudes y lenguajes discriminatorios e injustos.

Si se quiere estudiar, enseñar, investigar y "pedagogizar la paz", hay que "ampliar nuestra comprensión de la misma y clarificar cuál es su significación"6, lo que amerita resaltar algunas características al respecto, destacando que Johan Galtung introduce la idea de paz positiva a finales de la década de los sesenta del siglo $X X^{7}$. Posteriormente este concepto se ha enriquecido con varios aportes desde diferentes miradas ${ }^{8}$. Naciones Unidas, proclama el Decenio Internacional de una cultura de paz y no violencia para los/as niños/as del mundo, del 2001 al $2010^{9}$.

John Paul Lederach, plantea que "cuando consideramos la paz desde diferentes perspectivas - popular, cultural, lingüística, científica - comprendemos que la paz es un valor (uno de los más altos y deseados del vocabulario y de la reflexión humana), un ideal y un concepto rico y multi-dimensional: está relacionada con todos los niveles de la existencia humana"10. En el artículo 22 de la Constitución Política de Colombia de 1991, la paz se consagra como un derecho fundamental y un deber de obligatorio cumplimiento.

Desde la perspectiva de género, se destaca el concepto de paz invisible, que

[...] responde al empeño de dar visibilidad a algunas acciones y comportamientos concretos que pasan desapercibidos, a pesar de que constituyen elementos centrales de la "paz positiva" porque refuerzan la armonía social comunitaria. La invisibilidad de esta paz se debe, en parte, a que las mujeres son sus protagonistas en la cotidianidad. Irónicamente, la misma violencia estructural que acentúa las desigualdades relacionadas con el género para las mujeres, hace que exista una paz invisible en donde se contrarresta dicha violencia. ${ }^{11}$

5 Paulo Freire, Pedagogía del oprimido, trad. Jorge Mellado [1. era ed., 1970] (México: Siglo XXI, 2010), 69.

6 John Paul Lederach, El abecé de la paz y los conflictos. Educación para la paz (Madrid: Catarata, 2000), 178.

7 Johan Galtung, Tras la violencia, 3R: reconstrucción, reconciliación, resolución. Afrontando los efectos visibles e invisibles de la guerra y la violencia, trad. Teresa Toda (Bilbao: Red Gernika 6, Bakeaz y Gernika Gogoratuz, 1998).

8 Irene Comins Mingol, “Horizontes epistemológicos de la investigación para la paz: una perspectiva pazológica y de género", en Enfoques contemporáneos para los estudios de paz, ed. Dora Elvira García-González (México: CONACYT / Tecnológico de Monterrey, 2018), 49.

9 Naciones Unidas, Asamblea General, Resolución A/RES/53/25 19 noviembre 1998.

10 Lederach, El abecé de la paz, 178.

11 Teresa Langle de Paz, La urgencia de vivir. Teoría feminista de las emociones (Barcelona: Anthropos / México: Universidad Autónoma Metropolitana, Iztapalapa, 2018), 49. 
Con la expresión paz invisible, Teresa Langle hace "referencia a comportamientos y gestos espontáneos y cotidianos, intensidades y expresiones de afecto y alegría que brotan y germinan gracias a una tendencia inquebrantable del ser humano a buscar el 'bienestar'"12; para esta autora, "cuando la paz invisible germina y se fortalece, garantiza un contexto difícilmente reversible que lleva al empoderamiento y engendra liderazgos comunitarios"13. Esto se evidencia en el surgimiento de gran cantidad de organizaciones lideradas por mujeres, aun en medio de las guerras, como ha sido el caso de Colombia ${ }^{14}$.

Es importante resaltar este aspecto, ya que se considera que "la paz invisible ocurre cuando, a pesar de la adversidad o en la adversidad, existe también aquello que produce placer y unas aptitudes y mecanismos que generan 'bienestar' y son intrínsecos a este. Y es que el espíritu humano es imbatible, creativo, resiliente; capaz de imaginarse, reinventarse y crearse en la fenomenología del presente, además de proyectarse hacia el futuro"15, ejemplo de lo cual son las poblaciones que sobreviven en medio de circunstancias muy traumáticas y continúan con sus vidas.

Así, la resiliencia como fuerza transformadora, que en palabras de Francisco Jiménez y Andrea Barrientos, se entiende como "el camino o capacidad de sobreponerse a una adversidad, además, de salir fortalecido para poder seguir caminando en la vida"16, permite contemplar la potencialidad de los seres humanos para remontar las dificultades, llenarse de fuerza y coadyuvar a cambiar algunas situaciones con el fin de proseguir adelante con su derrotero. En este contexto, "la resiliencia conecta con la investigación para la paz, al considerar que los conflictos son la base del desarrollo humano"17, lo que es relevante porque no oculta la existencia de conflictos de distinta naturaleza, lo cual corresponde a la realidad de cualquier sociedad.

En esta línea, Lederach señala que hay que ver como se toman "[...] la noviolencia y el pacifismo como materias pedagógicas"18, asunto de transcendental importancia porque conlleva acciones propias de procesos emancipatorios y de empoderamiento, que cimientan propuestas educativas y pedagógicas con las comunidades y las instituciones educativas, como las expuestas a continuación:

La Pedagogía Multicultural Revolucionaria ${ }^{19}$, dinamiza e incrementa las teorizaciones y el debate sobre multiculturalismo; así emergen puntos de vista discordantes. El multiculturalismo revolucionario se enriquece con la mirada crítica,

\footnotetext{
12 Ibid.

13 Ibíd.

14 Cfr. Celina de Jesús Trimiño Velásquez, Las mujeres protagonistas en el proceso de paz. Aproximaciones al caso de Colombia (Tunja: Editorial UPTC, 2018).

15 Langle de Paz, La urgencia de vivir, 50-51.

16 Francisco Jiménez Bautista y Andrea Barrientos Soto, “Buscando cartografías de paces: la paz resiliente”, en Enfoques contemporáneos para los estudios de paz, ed. Dora Elvira García-González (México: CONACYT / Tecnológico de Monterrey, 2018), 98.

17 Ibíd.

18 Lederach, El abecé de la paz, 106-108.

19 Cfr. Peter McLaren y Ramin Farahmandpur, La Enseñanza contra el Capitalismo Global y el Nuevo Imperialismo. Una Pedagogía Crítica, trad. Néstor Cabrera (España: Editorial Popular, 2006).
} 
que da lugar a tendencias de inclusión democrática y no discriminatoria de los grupos humanos, demandando sociedades igualitarias. Esto se expresa en "[...] la pedagogía crítica, que - al menos en la tradición revolucionaria que [ellos defienden aquí] - es fuertemente antirracista, antisexista y antihomofóbica" ${ }^{20}$. Esto evidencia una articulación con la teoría de la interseccionalidad.

La Pedagogía del empoderamiento de grupos excluidos ${ }^{21}$, engarza con lo anterior. Desde la óptica de la interculturalidad y de la educación en derechos humanos, es importante potenciar grupos o personas que, tanto en la vida como en los procesos sociales, políticos, económicos, culturales, entre otros, han sido excluidas, negadas e invisibilizadas; así, a través de la toma de conciencia crítica que problematiza las relaciones sociales, se hace posible la promoción de procesos que favorezcan a grupos específicos históricamente discriminados.

La Pedagogía de la indignación, permea varias concepciones sobre la pedagogía y resalta el profundo nexo entre política y educación. “La cuestión fundamental de la práctica política no es simplemente hacer cosas, sino en beneficio de qué y de quién se hacen cosas, lo que, en cierto sentido, implica en contra de quién se hacen las cosas (...) Como no es posible separar la política de la educación, el acto político es pedagógico y el pedagógico es político" ${ }^{22}$, reflexiones que se constituyen en herramientas para el análisis del ejercicio de la política, la formulación de las políticas públicas, de la aplicación de la justicia y la consecución de una verdadera justicia social.

Esto se enlaza con la pedagogía antidiscriminatoria, teniendo en cuenta que en las sociedades contemporáneas permanecen y afloran una serie de discriminaciones por diferentes razones, que no pueden desligarse de las intersecciones, por ello "promover una pedagogía antidiscriminatoria supone una atención crítica hacia todos los aspectos destacados y exige romper el silencio, la invisibilización y la subordinación, denunciar la discriminación y desarrollar nuevas estrategias y prácticas de empoderamiento, reconocimiento y construcción afirmativa de las diferentes identidades" 23 , respetando, en todas las prácticas, la diferencia.

Aunque el tema de la memoria tiene antecedentes, entre ellos, el de la Europa de la segunda posguerra del siglo $\mathrm{XX}$, las experiencias en varios países muestran que en el marco de la educación en derechos humanos y para la paz, va adquiriendo vital importancia la educación y la pedagogía de la memoria ${ }^{24}$ en estrecha relación con la protección de los derechos de las víctimas. En el caso de Colombia, en el artículo 56 de la ley 975 de 2005, se plantea el “Deber de

20 Ibíd., 191.

21 Cfr. Susana Sacavino y Vera Maria Candau, Multiculturalismo, interculturalidad y educación: contribuciones desde América Latina (Bogotá: Ediciones desde abajo, 2015), 139-140.

22 Paulo Freire, Pedagogía de la indignación, trad. Tomás del Amo (Madrid: Morata, 2010), 140.

23 Sacavino y Candau, Multiculturalismo, 150.

24 Piedad Ortega Valencia et al., Pedagogía de la memoria para un país amnésico (Bogotá: Universidad Pedagógica Nacional, 2015); María Andrea Rocha Solano, "Pedagogía de la historia y las memorias en contextos políticos turbulentos", en Narrativas de experiencia en educación y pedagogía de la memoria, comp. Gabriel Jaime Murillo Arango (Buenos Aires: CLACSO / Editorial de la Facultad de Filosofía y Letras Universidad de Buenos Aires, 2015). 
memoria" ${ }^{25}$, y el artículo 142 de la ley 1448 de 2011 consagra el "Día nacional de la memoria y solidaridad con las víctimas. El 9 de abril de cada año, se celebrará el Día de la memoria y Solidaridad con las Víctimas" ${ }^{26}$, se conmemora desde el 2012. Más allá de lo anterior, Gabriel Murillo considera que "el ejercicio de la memoria histórica" ${ }^{27}$ no ha contado con espacios amplios en la escuela y no se ha desarrollado teniendo en cuenta su importancia en un país que ha mantenido un largo conflicto armado.

No obstante, algunas iniciativas lideradas por la academia, diversas organizaciones y sectores de la población, se han ocupado del tema. En este contexto, puede destacarse el trabajo desarrollado por el Centro Nacional de Memoria Histórica (CNMH), iniciado por el Grupo de Memoria Histórica $(\mathrm{GMH})^{28}$; el $\mathrm{CNMH}$ ha planteado varios proyectos en el área de Pedagogía.

Determinadas universidades en Colombia han iniciado el camino y se espera que otras avancen al respecto. Mención especial reviste el grupo de la Universidad Pedagógica Nacional, que considera que "[...] la comprensión y superación del pasado plantea la necesidad de otorgarle un lugar a la memoria en los procesos de formación en Derechos Humanos, entendiéndola como proceso social y político que tiene como centro la recuperación crítica de la historia y que posibilita la apropiación y comprensión del pasado, como clave para la transformación del futuro" ${ }^{29}$. Esto sugiere que la pedagogía de la memoria tiene en cuenta que la memoria se construye poniendo a dialogar a las personas sobre el presente, con las memorias del pasado para tomar decisiones con relación al futuro.

En todo esto, la escuela y la educación no pueden desconocer su rol fundamental desde los primeros grados del sistema educativo, puesto que la educación es libertad y como lo contempla la teoría freiriana, "Los niños precisan tener asegurado el derecho de aprender a decidir, cosa que sólo se hace decidiendo" 30 , así, hay que tener en cuenta el respeto por la niñez, por sus decisiones, deseos y particularidades, tomando como base que "la educación es un acto de amor, por tanto, un acto de valor" 31 , en el que las variables contextuales del aprendizaje de cada individuo son únicas y necesariamente deben ser tenidas en cuenta.

Así, dentro de este espectro de posibilidades, surgen los principios de la pedagogía parental, que evidencia cómo la familia hoy en día tiene una respon-

25 Congreso de la República de Colombia, ley 975/2005, de 25 de julio, por la cual se dictan disposiciones para la reincorporación de miembros de grupos armados organizados al margen de la ley, que contribuyan de manera efectiva a la consecución de la paz nacional y se dictan otras disposiciones para acuerdos humanitarios (Diario Oficial n. ${ }^{\circ} 45.980$, Bogotá: 2005), 30.

26 Congreso de la República de Colombia, ley 1448/2011, de 10 de junio, por la cual se dictan medidas de atención, asistencia y reparación integral a las víctimas del conflicto armado interno y se dictan otras disposiciones (Diario Oficial n. ${ }^{\circ} 48$ 096, Bogotá: 2011), 48.

27 Gabriel Jaime Murillo Arango, "Los trabajos y los días de una pedagogía de la memoria", en Narrativas de experiencia en educación y pedagogía de la memoria, comp. Gabriel Jaime Murillo Arango (Buenos Aires: CLACSO / Editorial de la Facultad de Filosofía y Letras Universidad de Buenos Aires, 2015), 314.

28 Rocha Solano, "Pedagogía de la historia", 341.

29 Ortega Valencia, et al., Pedagogía de la memoria, 139.

30 Freire, Pedagogía de la indignación, 71.

31 Paulo Freire, La educación como práctica de la libertad [1967] (Madrid: Siglo XXI, 2009), 67. 
sabilidad pasiva y un rol débil en cuanto al proceso educativo, pues esta tarea se ha destinado en su totalidad a la escuela; la pedagogía parental, propuesta por Augusta Moletto y Riziero Zucchi, aporta razones para darle importancia al rol de la familia en la educación. La familia cumple sus deberes educativos y responde por los mismos, enseña de una manera espontánea y en este trascurso de aprendizajes las madres y los padres de familia son las personas que conocen perfectamente a sus hijos e hijas, pero en la escuela la familia no aporta este conocimiento para que la maestra o maestro conozca los trayectos escolares de sus estudiantes. La familia posee recursos y competencias que deben ser reconocidas por la escuela, por lo tanto, la metodología de la pedagogía parental busca dignificar la acción pedagógica de los padres y las madres como agentes educativos expertos, por medio de iniciativas que promuevan sus conocimientos ${ }^{32}$. Para Moletto y Zucchi ${ }^{33}$, la Pedagogía dei Genitori se expresa a través de:

- La pedagogia della responsabilità: la famiglia adempie ai compiti dell'educazione e ne risponde al mondo.

- La pedagogia dell'identità: l'amore dei genitori fa sviluppare una consapevolezza che permette alla persona di riconocersi.

- La pedagogia della speranza: la speranza dei genitori e l'anima del progetto di vita, del pensami adulto.

- La pedagogia della fiducia: la fiducia della famiglia non solo sostiene le potenzialità del figlio, ma le fa nascere.

- La pedagogia della crescita: i genitori sono testimoni e attori del percorso di sviluppo del figlio.

Cabe anotar que la pedagogía parental propone un nuevo pacto educativo entre la escuela, familia e instituciones sociales, en el que madres y padres asumen un rol activo gracias al reconocimiento de sus competencias ${ }^{34}$, estos elementos deben tenerse en cuenta, pues la señalada pedagogía puede tener protagonismo en escenarios como el de Colombia. En otro escrito se ha planteado que en este país la mayoría de las personas "no han experimentado lo que significa vivir en paz"35, esto dificulta su comprensión, por lo tanto se deben aunar todos los esfuerzos orientados a reflexionar sobre la educación en derechos humanos y para la paz, haciendo que el proceso de construcción de una paz estable y duradera "sea prioridad para la población colombiana, generando una apropiación colectiva, con el fin de que la paz sea posible y se convierta en realidad"36.

En este panorama "[...] la educación no sexista para la paz le apuesta a la verdad, justicia y reparación frente a las formas de violencias contras las mujeres en el marco del conflicto social y armado en [Colombia]"37; se destaca,

32 Cfr. Augusta Moletto y Riziero Zucchi, La metodologia pedagogia dei genitori. Valorizzare il sapere dellesperienza (Bolzano: Maggioli Editore, 2013), 37

33 Augusta Moletto y Riziero Zucchi. "Pedagogia dei genitori”, en Per allevare un bimbo ci vuole un villaggio..., eds. Insegnanti e genitori del l'IPC (Bolzano: Intendenza Scolastica Italiana, 2012), 20.

34 Cfr. Augusta Moletto y Riziero Zucchi, La Metodologia Pedagogia dei genitori, 35-49.

35 Trimiño Velásquez y Amézquita Aguirre, "Reflexiones desde la universidad", 120.

36 Ibíd.

37 Diana María Montealegre, “¿Para qué hablar de una educación no sexista como contribución a la paz?”, en Una educación no sexista: 
"la importancia de iniciativas pedagógicas como la Comisión de la Verdad de las Mujeres de Colombia, liderada por la Ruta Pacífica de las Mujeres" ${ }^{\prime 38}$. En la siguiente parte, se argumentará que la perspectiva de género y el desarrollo de la interseccionalidad, aportan desde otros enfoques a las pedagogías para la paz.

\section{Géneros e interseccionalidad}

Pese al avance de las teorías, experiencias y discursos sobre la paz, en los debates se observa un tímido componente relacionado con los géneros y las intersecciones, cuyos planteamientos adquieren cada vez más importancia en los procesos de paz, pues su construcción requiere incorporar la diversidad de los seres humanos. Rita Segato nos recuerda, más allá de las amplias y fructíferas discusiones, “[...] que género no es otra cosa que una categoría analítica que pretende dar cuenta de cómo representaciones dominantes, hegemónicas, organizan el mundo de la sexualidad, de los afectos, de los roles sociales y de la personalidad" 39 , lo que se reproduce con distintos matices y en la realidad connota ciertas particularidades.

Bajo esta óptica hay que contemplar lo señalado por Langle: "aunque la mayoría de los relatos oficiales de la violencia y la paz ignoren la preponderancia del género como factor determinante y sistémico o la relativicen, las mujeres, por su género, muy a menudo son tan protagonistas de la violencia ejercida contra ellas como de la paz" ${ }^{40}$. Asimismo, hay que agregar que en algunos países ellas también participan activamente en las guerras o conflictos armados, sea como parte de la "fuerza pública" compuesta por las Fuerzas Militares y la Policía Nacional ${ }^{41}$, o como integrantes de los grupos alzados en armas, como ha ocurrido en Colombia, lo cual asimismo es expresado por Carolina María Gómez, quien subraya que en los años de mayor escalonamiento del conflicto en este país, también aumentó la participación de las mujeres como combatientes ${ }^{42}$.

En consonancia con lo expuesto, cuando Segato habla de masculinidad y de femineidad no habla "[...] de esencias, sino de historias diferentes: la historia de los hombres y la historia de las mujeres. Siempre considerando también la existencia de variaciones importantes con relación a lo que se entiende por un hombre y una mujer" 43 . Todo esto nos lleva a observar las realidades de las mujeres en cada país y lugar.

Un debate para la paz, ed. Mary Edith Murillo Fernández y María Andrea Simmonds Tabbert. (Popayán: Editorial Universidad del Cauca, 2018), 28.

38 Ibíd.

39 Rita Segato, Contra pedagogías de la crueldad (Buenos Aires: Prometeo, 2018), 26.

40 Langle de Paz, La urgencia de vivir, 50.

41 Constitución Política de Colombia de 1991, artículo 216, capítulo 7, de la Fuerza Pública.

42 Carolina María Gómez Fonseca, "Estudios de Género y Conflictos Sociales Armados", en Feminismos y poscolonialidad. Descolonizando el feminismo desde y en América Latina, comps. Karina Bidaseca y Vanesa Vazquez Laba (Buenos Aires: Ediciones Godot, 2011), 171.

43 Segato, Contrapedagogías, 41. 
De esta manera, en entornos de inseguridad en América Latina, y en el caso de Colombia,

[...] en los que además persiste la impunidad, la corrupción y la criminalización de líderes y liderezas (sic), las mujeres que defienden el territorio viven violencias específicas, que se articulan con las que históricamente hayan experimentado por razón de género, dado que sus cuerpos se transforman en otro territorio de explotación que es cosificado y apropiado para que sirva al capital. ${ }^{44}$

Todo lo cual ha sido denunciado por los feminismos, por organizaciones, entre ellas de mujeres, organismos y ONG nacionales e internacionales, y determinados medios de comunicación.

En este análisis se debe tener en cuenta que, en la idea de género subyace la categoría patriarcado, desarrollada por las teorías feministas e imprescindible para comprender este entramado socioeconómico y político. Desde este punto de vista, las sociedades patriarcales son entendidas como un sistema basado en un modelo masculino ${ }^{45}$, además como culturas que pretenden dar cabida solamente al binarismo hombre-mujer e imponer el heteropatriarcado, razón por la cual el mismo concepto de género no era suficiente y se ha problematizado a través de las polémicas planteadas. La idea de género se ha ido ensanchando desde las distintas corrientes de los feminismos, y en la actualidad se habla de géneros, en plural, para denotar la diversidad y posibilidades de ser y habitar en el mundo.

Por otra parte, aunque "hay diferencias significativas entre los países en la medida en la que ellos limitan a las mujeres, en la práctica originan la misma concepción de la mujer como individuo débil y privado, mientras que el hombre es la figura fuerte y política" 46 , a partir de lo cual "se generan dificultades en el acceso al proceso de toma de decisiones, al trabajo, a la política, a la participación social, a los grupos y al capital político. Esto es porque restringen el tiempo que tienen las mujeres para participar e informar sus acciones y decisiones, así como los espacios donde pueden participar" ${ }^{\prime 7}$. Desde esta mirada, pareciera que muchas sociedades patriarcales solo estuvieran compuestas de hombres, ignorando el cúmulo de conocimientos de las mujeres, sus experiencias y contribuciones al desarrollo de la humanidad en sus distintos aspectos.

En esta línea, Segato recuerda que "patriarcado y género son sinónimos por el tiempo que dure la prehistoria patriarcal de la humanidad [ella considera], que no hay evidencias documentales, ni históricas ni etnográficas, de otra forma de estar en el género que no sea patriarcal" ${ }^{\prime 4}$. No obstante lo anotado, permite

\footnotetext{
44 Ruth Vargas Rincón, comp. Mujeres en voz alta (Bogotá: Editorial Universidad Distrital Francisco José de Caldas, 2019 ), 82.

45 Eugenia Correa, "Sociedad patriarcal, las luchas por la equidad de género y el posneoliberalismo", en Feminismos para un cambio civilizatorio, coord. Alba Carosio (Caracas: Fundación Centro de Estudios Latinoamericanos Rómulo Gallegos; Centro de Estudios de la Mujer / Buenos Aires: CLACSO, 2014), 71.

46 Ibíd., 73.

47 Ibíd.

48 Segato, Contrapedagogías, 59.
} 
plantear que, si en los procesos de luchas se logran transformar estas estructuras, otras posibilidades se abrirán para todos los seres humanos. A partir de las prácticas y de relaciones de poder equitativas se podrán vivir y escribir otras historias de reconocimiento y respeto por todas las personas. Sociedades en las cuales las diferencias no signifiquen discriminación y aniquilación. Por el contrario, valorar esas diferencias y considerar a cualquier ser humano con igual dignidad, representaría un peldaño más en la historia de la humanidad, historia desde luego con matices, pero sin exclusiones odiosas.

Aunado a lo anterior, emergen otras categorías que tornan más complejo el estudio crítico de las sociedades patriarcales. Así, el término "interseccionalidad" fue argumentado por la feminista estadounidense Kimberlé Williams Crenshaw para visibilizar la situación de las mujeres negras ${ }^{49}$. Esta "teórica del derecho contra el racismo legal [realizó estos planteamientos en 1989] cuando discutió temas relacionados con el empleo de las mujeres negras en los Estados Unidos y la intersección de asuntos de género, raza y clase en su explotación y exclusión" ${ }^{\prime 50}$. En este sentido, para abordar el problema de la interseccionalidad, Crenshaw examina cómo los tribunales enmarcan e interpretan las historias de las mujeres negras demandantes ${ }^{51}$. La autora sugiere que en este marco no se puede tomar como un único eje la discriminación sexual, pues no se tendría en cuenta la discriminación racial y las experiencias de las mujeres negras, lo que también está atravesado por los privilegios de clase social ${ }^{52}$.

Crenshaw argumenta que, así, las mujeres negras muchas veces son excluidas de la teoría feminista y el discurso de las políticas antirracistas, pues no se incorpora la interacción entre raza y género, en otras palabras, no se considera la interseccionalidad ${ }^{53}$. Esta autora resalta que "la experiencia interseccional es mayor que la suma de racismo y sexismo, cualquier análisis que no tenga en cuenta la interseccionalidad no puede dimensionar suficientemente la manera particular en que las mujeres negras están subordinadas" ${ }^{54}$. Ellas a menudo experimentan la doble discriminación y los efectos combinados de las prácticas que discriminan por motivos de raza y basados en el sexo ${ }^{55}$. Así, el análisis del patriarcado no puede estar enraizado en la experiencia blanca ${ }^{56}$.

49 Kimberlé W. Crenshaw, "Demarginalizing the Intersection of Race and Sex: A Black Feminist Critique of Antidiscrimination Doctrine, Feminist Theory and Antiracist Politics", University of Chicago Legal Forum, vol. 1989, n. ${ }^{\circ} 1$ (1989): 138-167. http:// chicagounbound.uchicago.edu/uclf/vol1989/iss1/8

50 Nira Yuval-Davis, "Más allá de la dicotomía del reconocimiento y la redistribución: interseccionalidad y estratificación”, en La interseccionalidad en debate. Actas del Congreso Internacional: "Indicadores interseccionales y medidas de inclusión social en instituciones de educación superior", ed. Martha Zapata Galindo, Sabina García Peter y Jennifer Chan de Ávila, Berlín: Freien Universität Berlin, 2012, 22.

51 Cfr. Crenshaw, "Demarginalizing", 141.

52 Ibíd., 140.

53 Ibíd.

54 "Because the intersectional experience is greater than the sum of racism and sexism, any analysis that does not take intersectionality into account cannot sufficiently address the particular manner in which Black women are subordinated". Ibid.

55 Ibíd., 149.

56 Ibíd., 157 
Como se observa, al mismo tiempo que Crenshaw plantea la teoría de interseccionalidad, también la entrecruza con clase social, lo que amplía este concepto teniendo en cuenta los entornos socioeconómicos, políticos y culturales. Al respecto, Avtar Brah y Ann Phoenix, en el 2004, llaman la atención sobre "los complejos, irreductibles, variados y variables efectos que resultan cuando múltiples ejes de diferencia - económica, política, cultural, psíquica, subjetiva y experiencial- se intersectan en contextos históricos específicos" ${ }^{\prime \prime 2}$.

La raza y el género, como explica Segato, “[...] son creaciones históricas para la dominación, funcionales para la extracción de valor no reconocido, no remunerado - una plusvalía racial y patriarcal. Los productos y saberes que emanan de ciertos cuerpos, ya sea los cuerpos racializados o feminizados, son saberes y productos que tienen menos valor, que pueden ser apropiados por medio de una menor remuneración" ${ }^{\prime 58}$, lo cual ha sido una práctica generalizada y continúa vigente, como se observa en gran parte del mundo, aunque con matices. Desde luego, habría que profundizar a través de investigaciones, cómo se materializa en cada situación específica. Otras autoras consideran que “[...] tanto feminismos coloniales, como postcoloniales y decoloniales, examinan las maneras complejas en que el género interacciona con otros sistemas de opresión y discriminación, especialmente la etnia, la religión y la clase social" ${ }^{59}$.

En su disquisición, Nira Yuval-Davis deduce que "Interseccionalidad es un término metafórico, orientado a evocar imágenes de intersecciones de caminos, con un número indeterminado o disputado de éstos, dependiendo de los varios usuarios de los términos y de cuántas divisiones sociales son consideradas en el análisis interseccional particular. (...) esto puede cambiar considerablemente e ir de dos a una infinidad" ${ }^{60}$. Esta autora realiza una importante reflexión sobre "los límites del análisis interseccional y las categorías interseccionales" ${ }^{61}$, y presenta una interesante compilación de los aportes de diferentes autoras al respecto.

Así, en la actualidad se pueden evidenciar variadas intersecciones que además permiten un análisis multidimensional, que incluye las experiencias de las personas excluidas, vulneradas, etc., lo que sin duda es una contribución para los estudios en los campos social y educativo. En palabras de Riveros, "es esencial que el pensamiento feminista pueda dejar en evidencia las múltiples facetas que presenta la dominación" ${ }^{62}$, y que afecta a muchas personas, entre ellas, de forma concreta, a las mujeres.

57 Avtar Brah, "Pensando en y a través de la interseccionalidad", en La interseccionalidad en debate. Actas del Congreso Internacional: "Indicadores interseccionales y medidas de inclusión social en instituciones de educación superior", ed. Martha Zapata Galindo, Sabina García Peter y Jennifer Chan de Ávila, Berlín: Freien Universität Berlin, 2013.

58 Segato, Contrapedagogías, 59.

59 Ana Guil Bozal y Ruby Lisbeth Espejo Lozano, "Feminismos coloniales, post y decoloniales", en Historias de vida de maestras africanas y afrodescendientes. Reflexiones y contextos, comps. Diana Elvira Soto Arango, Martha Luisa Corbett-Baugh y Véronique Solange Ocome-Beka (Tunja: Editorial UPTC, 2020), 272.

60 Yuval-Davis, "Más allá de la dicotomía", 23.

61 Ibid., 25.

62 Clara Riveros, "Aproximaciones a los feminismos contemporáneos en América Latina”, en América Latina: una integración regional fragmentada y sin rumbo, coords. Consuelo Silva Flores, Ariel Noyola Rodríguez y Julián Kan (Buenos Aires: CLACSO, 2018 ), 198. 
En este sentido, Segato considera de "suma importancia mencionar (...) la otra faz del proceso histórico a través del cual el sujeto masculino se transforma en el sujeto representante de la Humanidad, es decir, deja de ser hombre para ocupar el pedestal de Hombre con mayúscula, es la minorización de todos quienes no coinciden con sus características" ${ }^{\prime 63}$, lo cual afecta a las mujeres y a variados sectores de la población, tanto en su visibilización, en su reconocimiento, como en el disfrute de sus derechos.

[...] la politicidad en clave femenina casi perdida se vuelve hoy más relevante que nunca porque estamos frente al derrumbe del canon político patriarcal. Ha quedado expuesta la ficción institucional. Urge entonces reencontrarnos y reatar los hilos con lo que perdimos en el camino de la historia: las maneras de las mujeres hacer y resolver la vida, la política en clave femenina que el monismo humanista enclausuró. ${ }^{64}$

Asunto transcendental porque implica reconocer la importancia de las experiencias de las mujeres y su papel, tanto en los ámbitos privados como públicos. Sus aportes serán cada vez más valiosos en los escenarios de participación y representación en la sociedad.

En este horizonte, Peter McLaren y Ramin Farahmandpur planteaban que "como marco para el desarrollo de una praxis pedagógica, el multiculturalismo crítico abre los espacios sociales y políticos para que los oprimidos desafíen las distintas formas de opresión de clase, raza y género que son producidas y reproducidas por las relaciones sociales dominantes" ${ }^{65}$, y realmente las poblaciones oprimidas han cuestionado el orden establecido y vienen afrontando una serie de retos encaminados a liderar cambios. Dentro de este panorama, se puede resaltar que el informe La verdad de las mujeres. Víctimas del conflicto armado en Colombia, elaborado por la Ruta Pacífica de las Mujeres ${ }^{66}$, es un gran aporte de las mujeres a los procesos de paz en este país, y se constituye en un referente obligado, si se quiere escuchar la voz y propuestas de amplios sectores de mujeres.

Asimismo, es necesario mencionar que las Organizaciones de la Sociedad Civil, "Las OSC de mujeres crearon alianzas efectivas para hacer incidencia por acuerdos sociales y políticas progresivas en el AFP [Acuerdo Final de Paz], dirigidas por la Cumbre de Mujeres (que consta de 9 redes de mujeres) y la Plataforma Cinco Claves (que consta de Sisma Mujer, Humanas y la Red Nacional de Mujeres)" ${ }^{67}$. Aquí se refieren al "Acuerdo final para la terminación del conflicto

\footnotetext{
63 Segato, Contrapedagogías, 66-67.

64 Ibíd., 67.

65 McLaren y Farahmandpur, La Enseñanza contra el Capitalismo, 145.

66 Ruta Pacífica de las Mujeres, La verdad de las mujeres. Víctimas del conflicto armado en Colombia, coord. Marina Gallego Zapata (Bogotá: G2 Editores, 2013).

67 ABColombia, "Hacia un Cambio Transformador: las mujeres y la implementación del Acuerdo de Paz colombiano", informe elaborado para la celebración del 2. ${ }^{\circ}$ aniversario del AFP, Londres: 2018. file:///C:/Users/Propietario/Desktop/Hacia-un-Cambio-Transformador-f-web.pdf
} 
y la construcción de una paz estable y duradera" ${ }^{68}$. Pese a las dificultades que persisten en Colombia, "El trabajo de las mujeres defensoras de derechos humanos sobre el enfoque de género en el Acuerdo de Paz y, más recientemente, en su implementación, ha elevado su visibilidad" ${ }^{69}$. En este sentido, lamentablemente hay que anotar que también se han convertido en un objetivo de los grupos contrarios a la paz, y varias de ellas hoy hacen parte del elevado número de personas asesinadas después de la firma del señalado acuerdo.

"A lo largo de la historia las mujeres han sido habladas y pensadas por los hombres, sin tener la posibilidad de hacerse oír y accionar con libertad"70. Las diferentes organizaciones de mujeres y los documentos producidos recogen las "epistemologías locales con las que se teje la historia universal. De ellas está hecha la Comisión de la Verdad de las Mujeres: conocimiento local femenino y feminista del impacto de la guerra en la vida y cuerpo de las mujeres" ${ }^{\prime \prime 1}$. Ellas nos enseñan en un "diálogo de saberes" otras formas de acceder a la verdad, de comprender la magnitud de la guerra, sus consecuencias, pero también de recomponer el tejido social.

Pese a la indignación y dolor por las vidas cercenadas, no se truncan las esperanzas de que en un país como Colombia se imagine la paz y se pueda transitar por estos senderos. En este marco, se incrementa la relevancia de la perspectiva de géneros y de la categoría intersecccionalidad, su desarrollo ayuda a profundizar los debates críticos y seguir trabajando en propuestas alternativas que involucren la educación, pero también al conjunto de la sociedad.

\section{Hacia unas propuestas alternativas}

De pensar un futuro de paz, justicia y libertad para las actuales y nuevas generaciones se infieren propuestas alternativas en el presente, sin olvidar, como afirma Lederach que "la educación para la paz es todo un proyecto, no sólo pedagógico sino también analítico, crítico y creativo"72, y en tal sentido el surgir de prácticas educativas y de alternativas pedagógicas hacen posible la promoción de futuros escenarios, que tengan en cuenta la diversidad de los seres humanos, uniendo así saberes para la paz. Por tanto, adquieren importancia los enfoques de género y las intersecciones.

De igual forma, tal como se evidencia, los planteamientos que surgen tienen en cuenta la resiliencia, sin olvidar que una persona resiliente opta por nuevas perspectivas de vida como artífice de su propia alegría y su propio destino, tal como lo argumentan Jiménez y Barrientos: "La resiliencia transciende el dolor,

68 Gobierno de la República de Colombia y Fuerzas Armadas Revolucionarias de Colombia-Ejército del Pueblo, "Acuerdo final para la terminación del conflicto y la construcción de una paz estable y duradera”, 24 de noviembre de 2016, Bogotá.

69 ABColombia, "Hacia un Cambio", 4.

70 Riveros, “Aproximaciones a los feminismos”, 197.

71 Socorro Corrales Carvajal, “Des-bordes femeninos en la educación: aportes a la paz", en Una educación no sexista: Un debate para la paz, ed. Mary Edith Murillo Fernández y María Andrea Simmonds Tabbert (Popayán: Editorial Universidad del Cauca, 2018 ), 64.

72 Lederach, El abecé de la paz, 177, 178. 
supera los traumas y transforma las crisis en posibilidades. Y esto nos lleva a dos realidades: a) adaptación, capacidad, superación y decisión, y b) afrontar la adversidad es salir fortalecido" 73 .

La intersectorialidad permite el confluir de caminos al centrar la mirada sobre las experiencias de las personas excluidas, vulneradas e invisibilizadas que, como lo menciona Magdalena Valdivieso, destaca la importancia de las desigualdades cruzadas, es decir que los planteamientos emergentes llevan a tener en cuenta "[...] como los sistemas de poder con base en género, raza, clase y sexualidad se apoyan mutuamente para producir exclusión, opresión y subordinación"74, donde la multiplicidad de aspectos enriquecen el aflorar de nuevos horizontes.

De esta manera, surgen algunas propuestas como las que se mencionan a continuación:

La articulación de currículos diferenciados, es decir, las acciones educativas que faciliten la disminución de discriminaciones por causa de sexo, raza, género o identidad sexual, etc.

[...] la composición del currículo escolar, tiene plena relación con la pedagogía intercultural en el aspecto de la selección de los conocimientos y saberes vehiculados (...). En este sentido es importante preguntarse y cuestionar los criterios que orientan la selección de contenidos y desconstruir las visiones monoculturales para abrirse al diálogo entre los diferentes conocimientos, saberes y culturas para dar lugar también a las producciones de los considerados otros, diferentes y siempre silenciados e invisibilizados. ${ }^{75}$

Hay que inquirir por otras ideas con el fin de reestructurar los parámetros establecidos desde arriba y explorar el mundo y la vida con lentes que abarquen el arcoíris de la humanidad en sus variados aspectos.

De igual forma, Sacavino y Candau resaltan la importancia de la formación docente y la composición del equipo escolar, en relación con una pedagogía anti-discriminadora, para que las educadoras y los educadores puedan hacer propuestas que involucren, desde la cotidianidad, los diferentes sectores que identifican las problemáticas del alumnado.

Es importante que los currículos de formación inicial y continuada incorporen contenidos relacionados con la educación en derechos humanos e interculturalidad que ayuden a comprender y valorar la diferencia sin discriminación. Que los educadores estén preparados para abordar los temas y situaciones que provocan discriminación, sin minimizarlos ni naturalizarlos. Ya sea una discriminación

73 Jiménez Bautista y Barrientos Soto, "Buscando cartografías", 98.

74 Magdalena Valdivieso Ide, "Otros tiempos y otros feminismos en América Latina y el Caribe", en Feminismos para un cambio civilizatorio, coord. Alba Carosio (Caracas: Fundación Centro de Estudios Latinoamericanos Rómulo Gallegos; Centro de Estudios de la Mujer / Buenos Aires: CLACSO, 2014), 28.

75 Sacavino y Candau, Multiculturalismo, 145-146. 
sexual, racial, de género o cualquier otra, debe ser enfrentada, desenmascarada y trabajada desde la óptica de la educación en derechos humanos, de afirmación de la dignidad y reconocimiento de la diferencia. ${ }^{76}$

Esta pedagogía anti-discriminadora facilitará hacer frente a todas las formas de discriminación y racismo, desde una actitud de la noviolencia y pacifismo, teniendo en cuenta que en todos los niveles de la educación es posible puntualizar componentes y formas de presentar la noviolencia como asignatura pedagógica. Por tanto, no solo bastará con la planeación teórica, sino que también será decisiva la actuación del profesorado y de toda la comunidad educativa.

Por lo tanto, la formulación de estrategias didácticas, metodológicas e innovadoras también favorece la formación de sujetos públicos críticos y sensibles a la solución pacífica de los conflictos, teniendo presente que, como afirma Belloso:

Construir la paz no significa, de ningún modo, tener amnesia. La construcción de la paz pasa por tener memoria. Pasa, asimismo, por ser capaces de construir un futuro a partir de esta memoria. Es necesario recordar, pero hay que recordar sin resentimientos. Y aunque muchas veces nosotros no seamos responsables de los males pasados, no quiere decir que no los reconozcamos, principalmente para no repetirlos. Para llegar a construir la paz, hemos de conocer la historia, pero hemos de transmitirla sin resentimiento, sin contagiar a las generaciones del presente las heridas del pasado. ${ }^{77}$

Lederach también se refiere a esto cuando advierte que "la educación para la paz tiene que dar una visión de la violencia amplia, multi-dimensional y lo más completa posible, de cara a los estudiantes, a fin de evitar la parcialidad de la imagen limitada y sensacionalista que suelen promover los medios de comunicación y las películas"78; es imprescindible profundizar para tener una mayor comprensión de esas violencias. Todo esto sin olvidar el empleo de materiales de apoyo, a los que hay que prestar especial atención para fortalecer procesos de diálogo y reconocimiento de quienes históricamente han sido minimizados e invisibilizados.

El material didáctico es otra fuente productora de invisibilización y discriminación que debe también ser analizado críticamente desde la óptica de la educación en derechos humanos, que rompa con la producción de jerarquías y desigualdades. Es importante tomar conciencia del tipo de contenidos, imágenes, valores, informaciones que son valorizadas y destacadas y cuales son silenciadas e invisibilizadas. Es muy frecuente encontrar materiales didácticos que refuerzan las visiones dominantes y los perjuicios en relación a las cuestiones étnicas, de género, de opción sexual, religiosas, de clase social, etc. Esta invisibilización, transformada en una

$76 \quad$ Ibíd., 149.

77 Nuria Belloso Martín, "La controvertida aparición de un nuevo derecho ciudadano: el derecho a la reparación de la memoria personal y familiar y la búsqueda de su justificación en la justicia transicional", en Conflicto armado, justicia y memoria, comp., Enán Arrieta Burgos (Medellín: Universidad Pontificia Bolivariana, 2016), 136.

78 Lederach, El abecé de la paz, 85. 
discriminación, contribuye para reforzar la formación de una autoestima negativa e inferiorizada de esos sujetos sociales. ${ }^{79}$

Si desde la misma etapa de planeación escolar se promueven y unen los saberes para la paz, paulatinamente, en las nuevas generaciones, las apropiaciones de los discursos permitirán evidenciar términos y actitudes igualitarias, de respeto y reconocimiento del otro y de la otra: para ello los preceptos freirianos de la educación dialógica, que se promueven desde una pedagogía concienciadora, donde la empatía, en definitiva, debe regir la comunicación dentro y fuera del aula, tal como se plantea "apelando al debate grupal sobre situaciones existenciales significativas para quienes se incorporaban al mundo de la lecto-escritura, en las que el sujeto debía ser activo, dialogante y desarrollar su espíritu crítico" ${ }^{80}$.

En el ámbito de la Pedagogía Social y la Educación Social, Fanny Añaños-Bedriñana categoriza varios Campos emergentes, dentro de los cuales se pueden resaltar la "[...] Educación intercultural, (...) Educación para la ciudadanía, Educación para la paz, Educación para la igualdad (género y otros colectivos)" que se encuadran en estas propuestas. Por demás, no cabe duda de que, en la acción educativa, no puede desconocerse la realidad directa de los educandos y las educandas, en todos los niveles; por ello, en Colombia se podría hablar de una educación en contexto, donde los procesos de migración interna, desplazamiento forzado, producto del conflicto armado y de las condiciones socio-económicas de inequidad y desigualdad, afectan tanto a las familias como a las instituciones educativas y comunitarias. A esto se suman las recientes migraciones que provienen de otros países, que también ponen en situación de vulnerabilidad a muchas personas:

\begin{abstract}
Este proceso de "movilización humana", que incluye de manera integral fenómenos diferenciados, en términos de conceptos especializados como migración, refugio, desplazamiento forzado, plantea múltiples temas desde la perspectiva de los derechos humanos. Esto incluye el origen frecuentemente convergente de tales procesos en conflictos armados internos o externos, y/o en la negación generalizada en las comunidades y los países de origen de condiciones dignas de vida que involucran violaciones serias de derechos económicos, sociales y culturales internacionalmente reconocidos, incluyendo la pobreza y la desigualdad, el hambre y la muerte o la discapacidad por enfermedades prevenibles. ${ }^{82}$
\end{abstract}

79 Sacavino y Candau, Multiculturalismo,146-147.

80 Abraham Magendzo-Kolstrein y María Isabel Toledo-Jofré, "Educación en derechos humanos: Estrategia pedagógica-didáctica centrada en la controversia", Revista Electrónica Educare, vol. 19, n. 3 (2015): 7. http://www.revistas.una.ac.cr/index.php/ EDUCARE/rt/printerFriendly/6864/7037 (20/11/2016).

81 "La PS es un campo de conocimiento pedagógico teórico, una disciplina científica - académica, una metodología... en constante redefinición en función de cada contexto, momento, necesidad, etc., que tiene como objeto de estudio una práctica educativa que denominamos Educación Social, ejercida fundamentalmente fuera del marco escolar, aunque en los últimos años se evoca y trabaja, también, en y desde la escuela" [Fanny T. Añaños-Bedriñana, "Pensamiento y acción socioeducativa en Europa y España. Evolución de la pedagogía y educación social”, Revista Historia de la Educación Latinoamericana, vol. 14, n. ${ }^{\circ} 18$ (2012): 133-135].

82 Camilo Pérez Bustillo, "Migración infantil, desplazamiento forzado, violencia estructural, conflicto y derechos humanos: marcos comparativos e implicaciones para Colombia y México, y sus contextos regionales" en Infancia y adolescencia en Colombia: tránsito hacia la paz, ed. Ernesto Durán (Bogotá: Observatorio sobre infancia / Universidad Nacional de Colombia, 2014), 64. 
En definitiva, es conveniente recalcar que una cultura de paz debe hacer parte de la educación y la formación ciudadanas, con lo cual es importante enfocar la paz como un "proceso creativo" y de resiliencia de manera que se fortalezca la construcción de una sociedad diversa, justa, igualitaria y democrática.

\section{CONCLUSIÓN}

A través de este artículo se han vislumbrado algunas epistemologías que permiten aproximarse a las pedagogías para la paz, en un ejercicio permanente que impregne el currículo y la vida cotidiana. Incardinado a lo anterior se muestra la relevancia de introducir otras miradas, incorporando el multiculturalismo crítico, la perspectiva de género(s) y la interseccionalidad. Este engarce constituye un aporte para dilucidar discriminaciones y avanzar en propuestas alternativas que se pueden enriquecer a partir de distintas disciplinas, pero también desde abajo. Esto implica un discurso pedagógico, analítico, crítico y creativo que facilitará el reconocimiento de las diferencias, el análisis y puesta en acción de planteamientos que comprendan las trayectorias y las resiliencias de las personas vulneradas e invisibilizadas. En consecuencia, las propuestas educativas y pedagógicas desde el prisma de la interseccionalidad, permiten que confluyan caminos para fortalecer los procesos emancipatorios en las comunidades y en las instituciones educativas, uniendo así saberes para la paz. Los enfoques de género(s) y de las intersecciones, además de permitir el análisis multidimensional, facilitan la construcción de un lenguaje y una actitud antirracista, antisexista y antihomofóbica, y una educación en contexto.

Las historias y experiencias de los grupos oprimidos son cada vez más importantes en las investigaciones y para las instituciones educativas y gubernamentales, pues partir del conocimiento situado contribuyen decididamente a generar teorías, a proyectar el plan de estudios y cualquier política teniendo en cuentas estas realidades. Por ello, comprender la paz como un valor hace que, en el ejercicio tanto de la práctica educativa, como en las acciones del diario vivir, se robustezcan los esfuerzos del conjunto de la sociedad colombiana para superar las violencias y contribuir a la consolidación de una paz estable y duradera. De esta manera, la promoción de currículos que implican la eliminación de discriminaciones odiosas por causa de sexo, raza, género, orientación sexual, clase social, etc., caracterizados por la formulación de estrategias didácticas, metodológicas e innovadoras de la formación de sujetos públicos críticos y sensibles, nutrirá el entramado para trabajar en la comprensión de los conflictos y la solución pacífica de estos, cuando sea el caso. 
Pensar en cómo construir la paz desde los contextos socioecónomicos y políticos de determinado país también implica vincular a la población, acercarse a sus condiciones y examinar las opresiones, lo cual exige una decisión colectiva para poner en práctica el pluralismo y la interculturalidad, todo lo cual significa apreciar la diversidad y la igual dignidad de todos los seres humanos, y en este sentido, defender sus derechos humanos tanto en las esferas privadas como públicas. Lo expuesto se contemplará en la programación y desarrollo de las asignaturas pedagógicas, desde su planeación hasta la generación del ambiente de toda la comunidad educativa, marcada por principios escolares donde se promuevan y articulen los conocimientos para la paz, desde los fundamentos de una pedagogía de la responsabilidad, de la esperanza, de la confianza, del crecimiento y antidiscriminatoria, que conduzca al empoderamiento de grupos marginados y de la recuperación de la memoria para la no repetición de distintos tipos de comportamientos que vulneran a las personas.

También se resalta que el empleo de materiales de apoyo, elaborados de forma cuidadosa y responsable, favorecerá los procesos de diálogo y el resarcimiento a amplios sectores, que históricamente han sido ignorados y vilipendiados. Así, paulatinamente, en las nuevas generaciones, las apropiaciones de los discursos permitirán evidenciar términos y actitudes de respeto de la otra y del otro, resaltando una pedagogía concienciadora en la que el sujeto activo y dialogante desarrolle su espíritu crítico. Por todo esto, una educación intercultural, una educación para la ciudadanía, para la paz, para la igualdad, beneficiará el trabajo conjunto con el propósito de transformar los entornos de las sociedades y abrir la ruta para reparar las injusticias infligidas a muchas personas excluidas por diversas razones, en Colombia y en otras latitudes del mundo.

\section{FUENTES}

Congreso de la República de Colombia. Ley 975/2005, de 25 de julio, por la cual se dictan disposiciones para la reincorporación de miembros de grupos armados organizados al margen de la ley, que contribuyan de manera efectiva a la consecución de la paz nacional y se dictan otras disposiciones para acuerdos humanitarios. Diario Oficial, n. ${ }^{\circ} 45$ 980, Bogotá, 2005.

Congreso de la República de Colombia. Ley 1448/2011, de 10 de junio, por la cual se dictan medidas de atención, asistencia y reparación integral a las víctimas del conflicto armado interno y se dictan otras disposiciones. Diario Oficial, n. ${ }^{\circ} 48$ 096, Bogotá, 2011.

Gobierno de la República de Colombia y Fuerzas Armadas Revolucionarias de Colombia-Ejército del Pueblo. "Acuerdo final para la terminación del conflicto y la construcción de una paz estable y duradera", 24 de noviembre de 2016, Bogotá, Colombia. 


\section{REFERENCIAS}

ABColombia. "Hacia un Cambio Transformador: las mujeres y la implementación del Acuerdo de Paz colombiano", informe elaborado para la celebración del $2 .^{\circ}$ aniversario del AFP, Londres: 2018. file://C:/Users/Propietario/Desktop/Hacia-un-Cambio-Transformador-f-web.pdf

Añaños-Bedriñana, Fanny T. “Pensamiento y acción socioeducativa en Europa y España. Evolución de la pedagogía y educación social". Revista Historia de la Educación Latinoamericana, vol. 14, n. ${ }^{\circ} 18$ (2012): 119 - 138.

Belloso Martín, Nuria. “La controvertida aparición de un nuevo derecho ciudadano: el derecho a la reparación de la memoria personal y familiar y la búsqueda de su justificación en la justicia transicional". En Conflicto armado, justicia y memoria [tomo 2: Derecho y transiciones hacia la paz], compilado por Enán Arrieta Burgos. Medellín: Universidad Pontificia Bolivariana, 2016, 115-152.

Brah, Avtar. "Pensando en y a través de la interseccionalidad". En La interseccionalidad en debate. Actas del Congreso Internacional: "Indicadores Interseccionales y Medidas de Inclusión Social en Instituciones de Educación Superior", editado por Martha Zapata Galindo Sabina García Peter y Jennifer Chan de Ávila. Berlín: Freien Universität Berlin, 2013, 14-20.

Comins Mingol, Irene. "Horizontes epistemológicos de la investigación para la paz: una perspectiva pazológica y de género". En Enfoques contemporáneos para los estudios de paz, editado por Dora Elvira García-González. México: CONACYT / Tecnológico de Monterrey, 2018, 45-68.

Constitución Política de la República de Colombia. http://www.secretariasenado.gov.co/ senado/basedoc/constitucion_politica_1991.html

Corrales Carvajal, Socorro. "Des-bordes femeninos en la educación: aportes a la paz". En Una educación no sexista: Un debate para la paz, editado por Mary Edith Murillo Fernández y María Andrea Simmonds Tabbert. Popayán: Editorial Universidad del Cauca, 2018, $55-71$.

Correa, Eugenia. "Sociedad patriarcal, las luchas por la equidad de género y el posneoliberalismo". En Feminismos para un cambio civilizatorio, coordinado por Alba Carosio. Caracas: Fundación Centro de Estudios Latinoamericanos Rómulo Gallegos; Centro de Estudios de la Mujer / Buenos Aires: Consejo Latinoamericano de Ciencias Sociales (CLACSO), 2014, 67-81.

Crenshaw, Kimberlé W. "Demarginalizing the Intersection of Race and Sex: A Black Feminist Critique of Antidiscrimination Doctrine, Feminist Theory and Antiracist Politics". University of Chicago Legal Forum 1989, n. ${ }^{\circ} 1$ (1989): 138-167. http://chicagounbound. uchicago.edu/uclf/vol1989/iss1/8

Freire, Paulo. La educación como práctica de la libertad [1967]. Madrid: Siglo XXI, 2009.

Freire, Paulo. Pedagogía del oprimido. Traducido por Jorge Mellado [1. ${ }^{\text {era }}$ ed. 1970]. México: Siglo XXI, 2010.

Freire, Paulo. Pedagogía de la indignación. Traducido por Tomás del Amo. Madrid: Morata, 2010.

Galtung, Johan. Tras la violencia, 3R: reconstrucción, reconciliación, resolución. Afrontando los efectos visibles e invisibles de la guerra y la violencia, traducido por Teresa Toda. Bilbao: Red Gernika 6, Bakeaz y Gernika Gogoratuz, 1998.

Gómez Fonseca, Carolina María. "Estudios de Género y Conflictos Sociales Armados". En Feminismos y poscolonialidad. Descolonizando el feminismo desde y en América Latina, compilado por Karina Bidaseca y Vanesa Vazquez Laba. Buenos Aires: Ediciones Godot, 2011, 165-177.

Guil Bozal, Ana y Ruby Lisbeth Espejo Lozano, "Feminismos coloniales, post y decoloniales". En Historias de vida de maestras africanas y afrodescendientes. Reflexiones y contextos, compilado por Diana Elvira Soto Arango, Martha Luisa Corbett-Baugh y Véronique Solange 
Ocome-Beka. Tunja: Editorial UPTC, 2020, 245-275.

Jiménez Bautista, Francisco y Andrea Barrientos Soto. “Buscando cartografías de paces: la paz resiliente". En Enfoques contemporáneos para los estudios de paz, editado por Dora Elvira García-González. México: CONACYT / Tecnológico de Monterrey, 2018, 85-109.

Langle de Paz, Teresa. La urgencia de vivir. Teoría feminista de las emociones. Barcelona: Anthropos / México: Universidad Autónoma Metropolitana, Iztapalapa, 2018.

Lederach, John Paul. El abecé de la paz y los conflictos. Educación para la paz. Madrid: Catarata, 2000.

McLaren, Peter y Ramin Farahmandpur. La Enseñanza contra el Capitalismo Global y el Nuevo Imperialismo. Una pedagogía Crítica, traducido por Néstor Cabrera. España: Editorial Popular, 2006.

Magendzo-Kolstrein, Abraham y María Isabel Toledo-Jofré. “Educación en derechos humanos: Estrategia pedagógica-didáctica centrada en la controversia". Revista Electrónica Educare 19, n. ${ }^{\circ} 3$ (2015): 1-16, http://www.revistas.una.ac.cr/index.php/EDUCARE/rt/printerFriendly/6864/7037 (20/11/ 2016).

Montealegre, Diana María. “¿Para qué hablar de una educación no sexista como contribución a la paz?". En Una educación no sexista: Un debate para la paz, editado por Mary Edith Murillo Fernández y María Andrea Simmonds Tabbert. Popayán: Editorial Universidad del Cauca, 2018, 17-30.

Moletto, Augusta y Riziero Zucchi. La metodologia pedagogia dei genitori. Valorizzare il sapere dell'esperienza. Bolzano: Maggioli Editore, 2013.

Moletto, Augusta y Riziero Zucchi. "Pedagogia dei genitori". En Per allevare un bimbo ci vuole un villaggio..., editado por Insegnanti e genitori del l'IPC. Bolzano: Intendenza Scolastica Italiana, 2012, 19-31.

Murillo Arango, Gabriel Jaime. "Los trabajos y los días de una pedagogía de la memoria". En Narrativas de experiencia en educación y pedagogía de la memoria, compilado por Gabriel Jaime Murillo Arango. Buenos Aires: CLACSO / Editorial de la Facultad de Filosofía y Letras Universidad de Buenos Aires, 2015, 313-335.

Naciones Unidas, Asamblea General, Resolución A/RES/53/25 19 noviembre 1998.

Ortega Valencia, Piedad, Clara Castro Sánchez, Jeritza Merchán Díaz y Gerardo Vélez Villafañe. Pedagogía de la memoria para un país amnésico. Bogotá: Universidad Pedagógica Nacional, 2015.

Pérez Bustillo, Camilo. “Migración infantil, desplazamiento forzado, violencia estructural, conflicto y derechos humanos: marcos comparativos e implicaciones para Colombia y México, y sus contextos regionales". En Infancia y adolescencia en Colombia: tránsito hacia la paz, editado por Ernesto Durán. Bogotá: Observatorio sobre infancia / Universidad Nacional de Colombia, 2014, 63-79.

Riveros, Clara. "Aproximaciones a los feminismos contemporáneos en América Latina". En América Latina: una integración regional fragmentada y sin rumbo, coordinado por Consuelo Silva Flores, Ariel Noyola Rodríguez y Julián Kan. Buenos Aires: CLACSO, 2018, 178-201.

Rocha Solano, María Andrea. "Pedagogía de la historia y las memorias en contextos políticos turbulentos". En Narrativas de experiencia en educación y pedagogía de la memoria, compilado por Gabriel Jaime Murillo Arango. Buenos Aires: CLACSO / Editorial de la Facultad de Filosofía y Letras Universidad de Buenos Aires, 2015, 337-368.

Ruta Pacífica de las Mujeres. La verdad de las mujeres. Víctimas del conflicto armado en Colombia. Coordinado por Marina Gallego Zapata. Bogotá: G2 Editores, 2013).

Sacavino, Susana y Vera Maria Candau. Multiculturalismo, interculturalidad y educación: contribuciones desde América Latina. Bogotá: Ediciones desde abajo, 2015.

Segato, Rita. Contra-pedagogías de la crueldad. Buenos Aires: Prometeo, 2018.

Soto Arango, Diana Elvira, José Pascual Mora García y José Rubens Lima Jardilino. "Formación de docentes y modelo pedagógico en la Universidad Pedagógica y Tecnológica 
de Colombia". Revista Historia de la Educación Latinoamericana 19, n. 29 (2017): 35-66. https://doi.org/10.19053/01227238.7552

Trimiño Velásquez, Celina de Jesús. Las mujeres protagonistas en el proceso de paz. Aproximaciones al caso de Colombia. Tunja: Editorial UPTC, 2018.

Trimiño Velásquez, Celina y Luisa Amézquita Aguirre. "Reflexiones desde la universidad sobre educación en derechos humanos y para la paz". Revista Historia de la Educación Latinoamericana 20, n. ${ }^{\circ} 31$ (2018): 101-124. https://doi.org/10.19053/01227238.8564

Valdivieso Ide, Magdalena. "Otros tiempos y otros feminismos en América Latina y el Caribe". En Feminismos para un cambio civilizatorio, coordinado por Alba Carosio. Caracas: Fundación Centro de Estudios Latinoamericanos Rómulo Gallegos; Centro de Estudios de la Mujer / Buenos Aires: CLACSO, 2014, 67-81.

Vargas Rincón, Ruth, comp. Mujeres en voz alta. Bogotá: Editorial Universidad Distrital Francisco José de Caldas, 2019.

Yuval-Davis, Nira. "Más allá de la dicotomía del reconocimiento y la redistribución: interseccionalidad y estratificación". En La interseccionalidad en debate. Actas del Congreso Internacional "Indicadores interseccionales y medidas de inclusión social en instituciones de educación superior", editado por Martha Zapata Galindo, Sabina García Peter y Jennifer Chan de Ávila. Berlín: Freien Universität Berlin, 2012, 21-34.

\section{Cómo citar:}

Amézquita Aguirre, Luisa; Trimiño Velásquez, Celina. “Pedagogías para la paz, la relevancia de la perspectiva de géneros y la interseccionalidad". Revista Historia de la Educación Latinoamericana. 22 No. 35 (2020): 65-86

https://doi.org/10.19053/01227238.11918

(1) (1) E Reconocimiento-No Comercial-Sin Obra Derivada 2.5 Colombia. 